\title{
Image Compression on Region of Interest based on SPIHT Algorithm
}

\author{
Sudeepti Dayal \\ Department of electronics and \\ communication \\ T.I.E.I.T \\ Bhopal, M.P., India
}

\author{
Neelesh Gupta \\ Department of electronics and \\ communication \\ T.I.E.I.T \\ Bhopal, M.P., India
}

\author{
Neetu Sharma \\ Department of electronics and \\ communication \\ T.I.E.I.T \\ Bhopal, M.P., India
}

\begin{abstract}
Image abbreviation is utilized for reducing the size of a file without demeaning the quality of the image to an objectionable level. The depletion in file size permits more images to be deposited in a given number of spaces. It also minimizes the time necessary for images to be transferred. Storage of medical images is most researched area in the current scenario. To store a medical image there are two parameters on which the image is divided, region of interest and non region of interest. The best way to store an image is to compress it in such a way that no important information is lost. Compression can be done in two ways namely, lossy and lossless compression. Under that several compression algorithms are applied. In the paper two algorithms are used that are, discrete cosine transform, applied to non region of interest (lossy) and discrete wavelet transform, applied to region of interest (lossless). The paper introduces SPIHT (set partitioning hierarchical tree) algorithm which is applied on the wavelet transform to obtain good compression ratio from which an image can be stored efficiently.
\end{abstract}

\section{Keywords}

Compression ratio, DWT, SPIHT, DCT

\section{INTRODUCTION}

One of the important aspects of image deposition is its efficient and effective abbreviation. An image, 1024 pixel $\mathrm{x}$ 1024 pixel x 24 bit, without any abbreviation, would need approximately $4 \mathrm{MB}$ of deposition space and 8 to 10 minutes for transmission, by employing a high speed, $68 \mathrm{Kbit} / \mathrm{s}$, ISDN line. If the image is compressed at 10:1 of compression ratio, the deposition requirement is lowered to $400 \mathrm{~KB}$ and the transmission time comes down to below 8 seconds. Seven 2 MB images can be compressed and transferred to a floppy disk in a very less time comparatively takes to transfer one of the original files. In a distributed environment, big image files still remain a major concern within systems. Compression is an important and beneficial technique for creating file sizes of manageable and transmittable dimensions. Increasing the bandwidth is another suitable method, but due to its cost sometimes makes less attractive or demanding solution. At the existing state of technology, the only outcome is to compress multimedia data before its deposition and transmission, and de-compress it at the receiver terminal for play back. For example, with a compression ratio of 32:1, transmission time requirements, the space and bandwidth can be reduced by a factor of 32, with acceptable quality. A common characteristic feature of most of the images is that the adjoining pixels are associated and therefore consists of redundant information.
The foremost task then is found to be less correlated representation of the image.

\subsection{Different Classes Of Compression Techniques}

\subsubsection{Lossless compression}

Under this type of compression arrangement, the rebuilt image, after compression, is numerically similar to the real image. However lossless compression can only access a modest amount of compression. An image rebuilt following lossy compression consisting degradation relative to the real. Often this is because of the compression arrangement completely discards redundant information.

\subsubsection{Lossy compression}

However, lossy patterns are capable of obtaining much higher compression. Under standard regarding conditions, no visible loss is recognized (visually lossless).

The information loss in lossy coding gets from quantization of the data. Quantization can be explained as the procedure of sorting the data into various bits and representing each bit with a value. The value selected to represent a bit is called the reconstruction value. Every item in a bit has the similar reconstruction value, which leads to the information loss (unless the quantization is so fine that every item gets its own bit).

\section{MEDICAL IMAGING}

With the fast growing technology in image compression some researchers extracted some advantageous and fruitful methods of storing medical images digitally. They realized that number of digital data can be stored further in the computer by compressing the original data or an image so that no essential information is lost and the size gets reduced without affecting the quality of an image. They further subdivided an image into two categories namely:

\section{ROI (Region of interest)}

2. Non-ROI

Medical Imaging is the technique of generating visual illustrations of the body for the medial analysis. Medical imaging uses various techniques such as MRI (magnetic resonance imaging), CT scan (computed tomography), X-ray, radiography, ultrasonography, endoscopy, electrocardiography and many more. These techniques are used to provide the area needs to diagnose. 


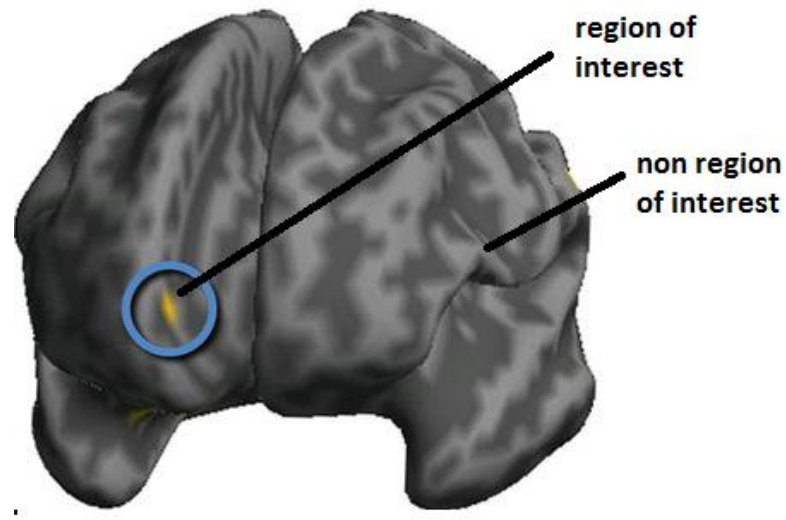

Fig 2: region of interest and non region of interest

\subsection{Region of interest}

All the information in a medical image is not equally important.ROI describes the affected part of an image and which has to be analyzed. This region is compressed so that reduced size of an image can be achieved with no information being lost. The ROI region is compressed using DWT (discrete wavelet transforms).

\subsection{Non region of interest}

In the process of compression the non ROI region is compressed more so that if there is any loss in information also it does not leads to any issue.

The non region of interest region is compressed using discrete cosine transform (DCT).

\section{SPIHT ALGORITHM}

Set partitioning in hierarchical trees is a based on wavelet which is very fast and come among the best image compression algorithm that offers fast execution time, good compression ratio and good image quality. This algorithm is applicable to lossless compression only.

The wavelet transformed image is of $16 * 16$ blocks (say) are divided into $8 * 8$ block with low and high intensities. The first block of the divided block is further sub divided into $4 * 4$ block. The figure below shows the arrangement.

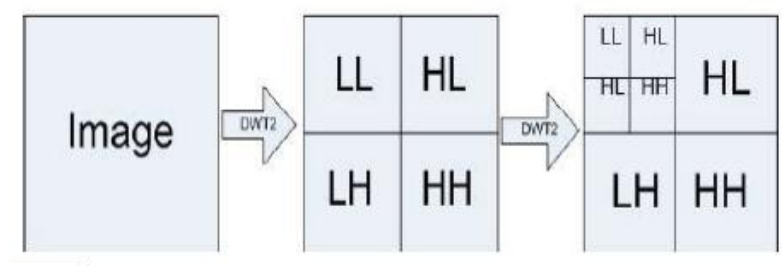

Fig 2: SPIHT process

\subsection{SPIHT parameters}

\subsubsection{Image quality}

Since wavelet transform outturn a good image quality. But when wavelet is combined with SPIHT not only produces good quality but also enhances the efficiency of an image. It can be said that SPIHT is a one step forward algorithm to wavelet encoders.

\subsubsection{Image transmission}

High quality image usually takes longer time to transmit the image in digital communication. Higher the quality of an image, larger will be the size. SPIHT is designed for excellent progressive transmission.

\subsubsection{Encoding and decoding speed}

The encoding and decoding time normally vary with CPU's. The algorithm takes approximately same time to encode and to decode irrespective of the processor.

\subsection{Analysis of SPIHT}

The analysis of the SPIHT algorithm starts with the wavelet decomposition which is applied on the image. The LL band which is shown in the figure 2 consists of the low pass coefficients. The other three bands consist of the vertical, horizontal and diagonal features of an image. The coefficients in the wavelet decomposition are assigned in a structured tree. The three lists are used to store the significant information during set partitioning. Those are:

List of insignificant pixels (LIP)

List of significant pixels (LSP)

List of insignificant sets (LIS)

One of the drawback faced in simple SPIHT algorithm is the slow processing speed which was overcome by modified algorithm called block based pass parallel SPIHT. In the modified algorithm first threshold value is set to begin with the analysis. Then all the coefficients of the wavelets are checked on column or row basis depend upon the user. If there is any pixel value in the row that is exceeding the threshold value then check the first half of the row values and then go for the next half. If no value greater than the threshold is found, proceed with the next matrix. The value of the threshold becomes half for every matrix. In this way every row and every column is check for every matrix. The algorithm is easy to explain and implement.

Threshold value $=\log 2(\max$ pixel value $)$

\begin{tabular}{|c|c|c|c|c|c|c|c|}
\hline 62 & 34 & 18 & 17 & -4 & 1 & -2 & 6 \\
\hline-31 & 24 & -15 & 14 & -11 & 0 & 4 & -1 \\
\hline \hline 42 & 20 & -35 & 10 & 29 & 10 & 6 & 9 \\
\hline-12 & 15 & -9 & 15 & -1 & 9 & 5 & 13 \\
\hline 4 & 45 & 13 & -1 & 26 & -21 & 3 & 1 \\
\hline 3 & 0 & -2 & -21 & -1 & 0 & 7 & 9 \\
\hline 0 & 13 & 4 & 5 & 4 & 5 & 6 & 0 \\
\hline-1 & 7 & -11 & 3 & 0 & 8 & 2 & 7 \\
\hline
\end{tabular}

Fig 3: analysis of SPIHT

As depicted in the above figure the maximum pixel value is 62 , therefore

Threshold value $=\log 2(62)=5$

\section{PROPOSED METHOD}

In this method an image has been taken and then segmented the image into region of interest (ROI) and non region of interest (non ROI) using seeded region growing method. The algorithm used to compress non ROI is discrete cosine transform and the algorithm used to compress ROI is discrete wavelet transform. After which SPIHT (Set partitioning in hierarchical trees) is applied to the wavelet. The algorithm is applied to a one dimensional image. Therefore they are reconstructed to get back the images in two dimensional. The 
ROI and non ROI images are combined which results in compression.

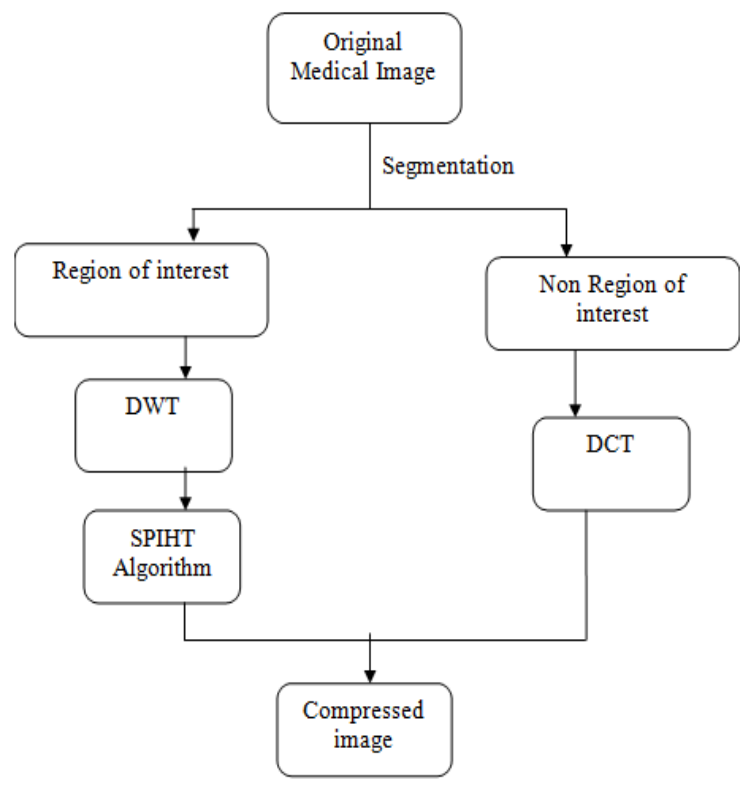

Fig:. 4 Block diagram of the proposed method

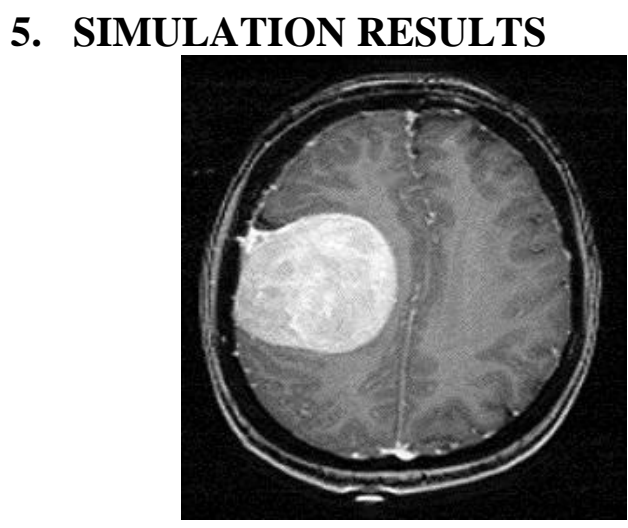

Fig: 5 Original image

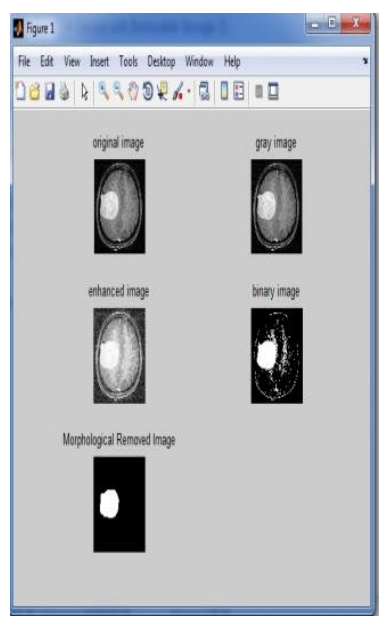

(a)

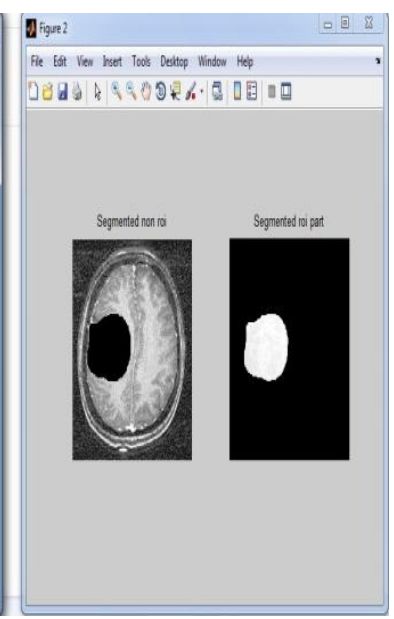

(b)
Fig: 6(a) Conversion of original image to greyscale and binary, (b) Segmentation of image.
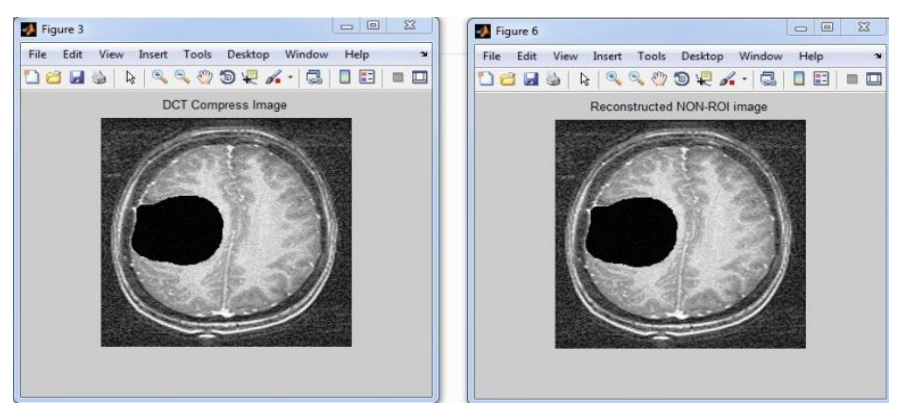

Fig: 7 DCT compressions applied to non-ROI and its reconstruction

Table1. Comparison between reference and the proposed work

\begin{tabular}{|c|c|c|}
\hline Parameter & $\begin{array}{c}\text { Reference } \\
\text { (ROI) }\end{array}$ & $\begin{array}{c}\text { Proposed } \\
\text { Method } \\
\text { (ROI+ SPIHT) }\end{array}$ \\
\hline Compression Ratio & 4.1134 & 4.6874 \\
\hline PSNR & 54.3964 & 58.97 \\
\hline MSE & & \\
\hline & 0.263 & 0.0825 \\
\hline
\end{tabular}

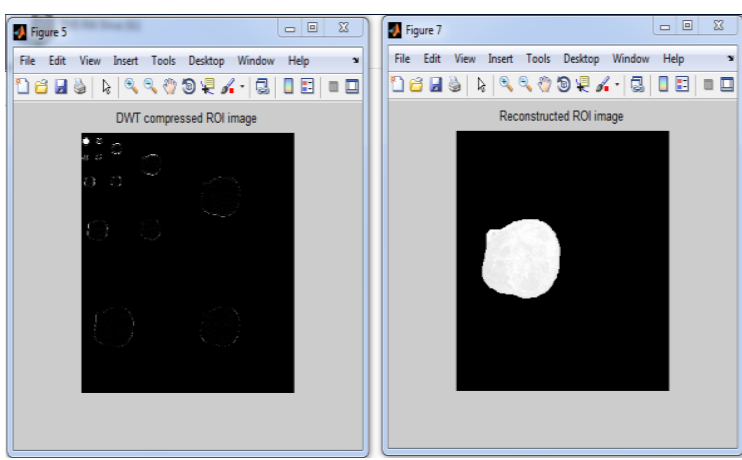

Fig.8 DWT compression applied to ROI and its reconstruction

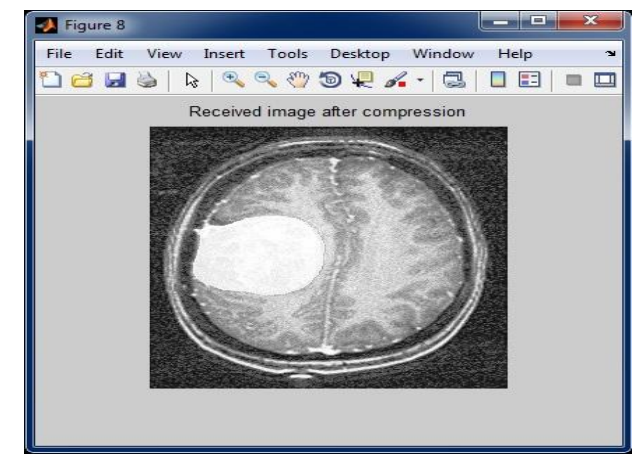

Fig: 9 compressed image 


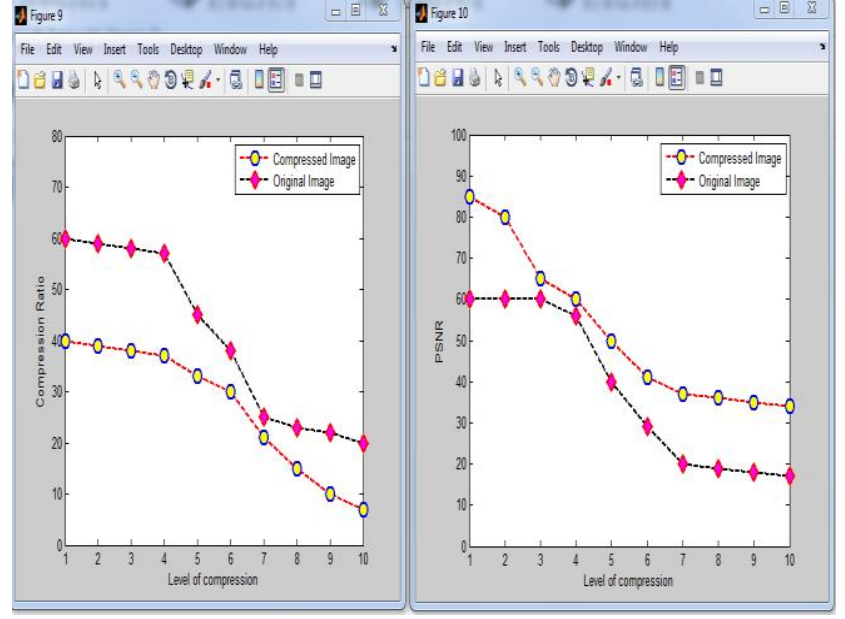

Fig: 10 Graph of compressed image to the original image in terns of PSNR and compression ratio

\section{CAlCUlationS}

In the proposed methodology the quality of an image is improved with the help of the parameters such as mean square error [MSE], peak signal to noise ratio [PSNR], compression ratio.

Mathematically these parameters are represented as:-

\section{Peak signal to noise ratio (PSNR)}

Psnr is the parameter for calculating the quality of a compressed image.

\section{$\mathrm{PSNR}=10 \log \left[\mathrm{i}^{\wedge} 2 / \mathrm{MSE}\right]$}

Where I= intensity of the image pixel level. For 5 bit per pixel, $I^{\wedge} 5-1=31$

\section{Compression ratio}

Compression ratio

Number of bits required for an original image Number of bits required for compressed image

\section{CONCLUSION}

In the paper develops the method for line dependent wavelet transforms. The paper focused on this transform that can be applicable to the encoder or the decoder and such that it can hold abbreviated form of data along with that, described highly scalable spiht coding algorithm which can operate with very less memory in addition to the combination with the linebased transform, and proved that its performance can be combative with condition of the art image coders, at a fragment of their memory utilization. The paper compares the performance of the reference paper [12], where results were carried out by applying discrete wavelet transform, and the proposed work, where SPIHT is applied to DWT (ROI), and concluded that the combination of wavelet transform and SPIHT Algorithm improves the compression ratio as well as PSNR and MSE. The work introduces a detailed execution of a low memory wavelet image coder. Its main significant and efficient advantage is that by making a wavelet coder that should be fair enough both in terms of speed and memory requirements.

\section{REFERENCES}

[1] C. Chrysas and A. Ortega, Line Based Reduced Memory Wavelet Image Compression," in Proc. IEEE Data Compression Conference, (Snowbird, Utah), pp. $398\{407,1998$

[2] W. Pennebaker and J. Mitchell,, JPEG Still Image Data Compression Standard. Van No strand Reinhold, 1994.

[3] D. Lee, New work item proposal: JPEG2000 image coding system." ISO, IEC JTC1, SC29, WG1 N390, 1996.

[4] J. M. Shapiro, "embedded image coding using zero trees of wavelet coefficients." IEEE Trans. Signal Processing, vol. 41, pp. 3445 3462, December 1993.

[5] Habibollah danyoli and Alfred mertins .Highly scalable image compression based on spiht for network applications

[6] Rafael C.Gonzalez, Richard E.Woods, Digital Image Processing, Prentice-Hall, 2002.

[7] Rafael C.Gonzalez, Richard E.Woods, Steven 1.Eddins, Digital Image Processing Using MATLAB, Prentice-Hall, 2004.

[8] A. M. Tekalp, Digital Video Processing. Englewood Cliffs, NJ: Prentice-Hall, 1995.

[9] M. Antonini, M. Barlaud, P. Mathieu, and I. Daubechies, "Image coding using wavelet transform," IEEE Trans. Image Processing, vol. 1, pp. 205-220, Apr. 1992.

[10] D. Taubman and A. Zakhor, "Multirate 3-D subband coding of video," IEEE Trans. Image Processing, vol. 3, pp. 572-588, Sept. 1994.

[11] Rolf Adams and Leanne Bischof, "Seeded region growing," IEEE transactions on pattern analysis and machine intelligence, vol. 16, no. 6, June 1994

[12] Mr. S.M. Kulkarni, Dr. D. S.Bormane, Dr. S.L.Nalbalwar, "Region of interest based coding technique applied to CT and MRI images for medical image compression," ELSEVIER.

[13] Sudeepti Dayal, Neelesh Gupta, Neetu Sharma, "Image compression using super resolution technique-A Review, " International Journal of Computer Applications (0975 - 8887) Volume 109 - No. 7, January 2015

[14] M. Suryanarayana Murthy, "Efficient Digital Image Compression by Using SPIHT Algorithm Combined with Huffman Encoding," International Journal of Engineering Research \& Technology (IJERT), Vol. 2 Issue 12, December - 2013. 